\title{
The European Diploma in Paediatric Radiology: a candidate's experience
}

\author{
Michael Paddock ${ }^{1,2}$ (1)
}

Received: 20 October 2021 / Revised: 20 October 2021 / Accepted: 31 October 2021 / Published online: 29 January 2022

(c) The Author(s), under exclusive licence to Springer-Verlag GmbH Germany, part of Springer Nature 2021

\section{Dear Editors,}

In 2017, the European Society of Paediatric Radiology (ESPR) launched the first cycle of the newly created European Diploma in Paediatric Radiology (EDiPR) [1]. This qualification was created to enable radiologists to further their subspecialist education and knowledge in paediatric radiology, and as a starting point to achieve "transnational harmonisation of paediatric radiologic standards in Europe" [2].

The International Pediatric Radiology (IPR) Congress 2021, held as a hybrid virtual and onsite conference in Rome, Italy, marked the first sitting of the final EDiPR examinations. Sixteen candidates from countries including Bulgaria, Czech Republic, Greece, Hong Kong, Ireland, Portugal, Qatar, Slovenia, Spain, Sweden, the Netherlands, and the United Kingdom - representing a truly international candidature - passed multiple examinations and met all the necessary requirements to be awarded the EDiPR.

In this editorial, the pathway and requirements of this qualification are outlined alongside my own experiences of having recently successfully completed the inaugural sitting of this diploma. It is hoped that this will encourage others, in particular junior radiologists training in paediatric radiology, to undertake this qualification alongside promoting the educational activities of the ESPR.

An overview of the diploma has been published previously [1]. Potential candidates should read the full EDiPR format, curriculum and eligibility information on the ESPR website $[2,3]$. By way of an overview, in order to satisfy

Michael Paddock

michael.paddock@doctors.org.uk

1 Medical Imaging Department, Barnsley Hospital NHS Foundation Trust, Gawber Road, Barnsley S75 2EP, UK

2 Department of Oncology \& Metabolism, University of Sheffield, Sheffield Children's NHS Foundation Trust, Sheffield S10 2TH, UK the demands of the educational curriculum and enter the final EDiPR examinations, candidates must complete the following:

- Three European Courses in Paediatric Radiology (ECPR) organised annually by the ESPR covering musculoskeletal imaging including systemic diseases, chest imaging including neck and cardiovascular, and abdominal imaging.

- Module 1 of the European Course in Paediatric Neuroradiology (ECPNR), a course jointly organised by the European Society of Neuroradiology (ESNR) and the ESPR (any module of the ECPNR was exceptionally accepted for the 2021 sitting but only Module 1 will be accepted thereafter).

My journey to the EDiPR commenced in 2017 with the musculoskeletal ECPR in Utrecht, the Netherlands, followed by the chest and abdominal ECPR in Dublin, Ireland, and Utrecht once again, in 2018 and 2019, respectively. Each course lasted 2 to 3 days (except Module 1 of the ECPNR, which lasted 5 days and was undertaken in Rome, Italy, in 2020) and comprised a series of lectures mapped to the European Society of Radiology level I to III training curriculum in paediatric radiology. The courses were held in comfortable venues and were very well catered with a plentiful supply of coffee during the breaks - a must for all radiologists! Each course concluded with a written examination on the last day and was either handwritten or taken electronically depending on the venue/location. In the case of an unsuccessful attempt at any course examination, candidates must wait until the next available ECPR cycle. All courses and examinations were conducted in English.

Whilst the most recent 2021 musculoskeletal ECPR was held virtually due to COVID-19 international travel restrictions, future courses are expected to be held in person to allow like-minded paediatric radiologists to meet, share experiences and facilitate networking. A significant 
advantage and attraction of these courses is that they are hosted in wonderful European cities that can be explored outside of the course. (A tip for future attendees: given that the ECPR tend to be held in early October, candidates will not be short of a crisp, autumnal setting when out and about for which warm clothing, including hats, gloves and scarves, is strongly recommended!)

I have a long-standing interest in paediatric neuroradiology and I elected to also undertake Module 2 of the ECPNR virtually in 2021 to further my knowledge and understanding of advanced paediatric neuroimaging following successful completion of Module 1. The particular strength of the ECPNR courses is the breakout seminars and workshops that facilitate discussion of clinical cases and reinforce learning from the preceding didactic lectures. Following successful completion of both examinations, I achieved the certificate of the ECPNR, which is "awarded to all doctors, regardless of individual specialty, who pass all two sets of the courses' written examination" [4] by the ESNR and the European Board of Neuroradiology (EBNR).

An exciting recent development is that holders of the EDiPR now partially satisfy the entry requirements to sit the European Diploma in Paediatric Neuroradiology (EDiPNR) examinations, whereas, candidates previously would have had to have held the European Diploma in Neuroradiology (EDiNR) in order to be eligible. The other eligibility criteria to enter the final EDiPNR examinations are found on the EBNR website [4].

I was extremely grateful to have been the beneficiary of several ESPR Junior Grants over the past 4 years, which allowed me to attend the required ECPR to fulfill the EDiPR requirements. This fantastic initiative is administered by the ESPR board "to provide financial support to eligible radiologists in training (residents/fellows) or young radiologists active in the field of paediatric radiology" [5, 6]. I would encourage all eligible radiologists to apply. Written confirmation of radiology training will be required, and applicants must be 40 years old or younger on the first day of the course. Funds will be paid to successful grant recipients after confirmation of attendance has been received by the ESPR following the respective ECPR.

As outlined above, to enter the final EDiPR examinations, candidates must have completed the required three ECPR courses and Module 1 of the ECPNR, and successfully passed all four written examinations. Moreover, candidates must be certified radiologists in their own country of practice (according to their national regulations); demonstrate evidence of training and/or practice in the field of paediatric radiology, be ESPR members in good standing at the time of application and have sufficient English language ability [3].

The first sitting of the final EDiPR examinations was originally scheduled for June 2020 at the 56th Annual ESPR Meeting in Marseille, France: unfortunately, both were cancelled due to the COVID-19 pandemic. They were rescheduled for the $8^{\text {th }}$ Conjoint IPR 2021 Congress in Rome, Italy. Given the hybrid nature of the conference (due to international travel restrictions and the potential changing global landscape), the examinations comprised a fully online multiple-choice question (MCQ) paper, and either an onsite (if the candidate was able to attend the IPR 2021 in person) or virtual oral examination. Only candidates who passed the final written examination were able to enter the oral examination. Only those who passed both the final written and oral examinations were awarded the EDiPR: candidates who fail the oral examination must retake both the written and oral components.

The online written examination took place before the start of IPR 2021 and was organised by the ESPR Education Committee in collaboration with the European Board of Radiology, using dedicated software to display the MCQs. The process was proctored live and recorded via proctoring software (downloaded as a plug-in extension to a compatible web browser) and also via a separate app on a compatible tablet/mobile device to monitor the room in which the examination took place. The examination was comprised of questions which were divided equally across the four components of the EDiPR curriculum (imaging of the brain and spine; chest, neck, and cardiovascular system; abdomen; and musculoskeletal system). At the time of IPR registration, the national picture with respect to the COVID-19 pandemic in the United Kingdom was challenging with significant restrictions still in place. As such, I elected to complete the oral examination virtually once I had received confirmation of having passed the written examination.

The oral examination (viva voce) was 35 minutes in length and comprised a series of clinical cases, each with multiple images across several body systems and imaging modalities. All relevant positive/negative (abnormal/normal) imaging findings were discussed, alongside differential diagnoses, the most likely diagnosis and appropriate subsequent management. The examination was conducted by two examiners -- in my case, a paediatric radiologist and a paediatric neuroradiologist, both of whom were eminently respected and internationally renowned professors, which was somewhat intimidating when they first appeared on the screen! However, they were both incredibly friendly and I really enjoyed the experience as it gave me the opportunity to demonstrate my knowledge and passion for paediatric radiology through a lively and interesting case discussion.

The results of both the written and oral examinations were released the following day, which was an incredibly quick and impressive turnaround for a postgraduate professional examination, much to my (and, I am sure, the other candidates') delight.

The EDiPR certification is valid for 5 years. Diplomats will be able to automatically renew their qualification 
through the ESPR by maintaining and meeting the following requirements within the 5-year period following the award:

- Member of the ESPR in good standing.

- Attendance at a minimum of two annual courses/congresses dedicated to paediatric radiology, e.g., ESPR, SPR, Asian and Oceanic Society for Paediatric Radiology (AOSPR), Sociedad Latinoamericana de Radiología Pediátrica (SLARP), IPR.

- One hundred continuing medical education/professional development (CME/CPD) points in relevant paediatric radiology activities.

I will use the EDiPR as a platform to encourage best practice in paediatric imaging and to promote our incredibly rewarding specialty to future generations of training radiologists.

At its heart, the EDiPR is a "measure of excellence" [1] demonstrating knowledge, competence and certification in paediatric radiology and represents a significant achievement in subspecialist paediatric radiology education across Europe to which all paediatric radiologists should aspire. I feel privileged to have been part of the inaugural cohort to have successfully completed this prestigious qualification. The courses provide opportunities to meet and socialise with other candidates who will go on to take responsibility for the promotion and potential evolution of our subspeciality in their place of work and individual country. Finally, the ESPR Education Committee should be commended for persevering with the organisation and administration of the ECPR and the final EDiPR examinations during such challenging international circumstances.

Acknowledgements The author is grateful to Dr Maria Raissaki, Chair of the ESPR Education Committee, for her insights in the preparation of this manuscript.

\section{Declarations}

Conflicts of interest Dr Michael Paddock is a member of the ESPR Education Committee. He has been included in discussions about the technicalities regarding the organisation of the examinations but was excluded from all communications regarding test material. He has been the recipient of several ESPR Junior Grants to support attendance at previous ECPR and ESPR annual meetings prior to joining the ESPR Education Committee.

\section{References}

1. Stafrace S, Nievelstein RAJ, Raissaki M, European Society of Paediatric Radiology Education and Professional Development Committee (2018) The European Society of Paediatric Radiology launches European Diploma in Paediatric Radiology. Pediatr Radiol 48:1040-1042

2. Education Committee (2021) European Diploma in Paediatric Radiology (EDiPR). European Society of Paediatric Radiology. Available at https://www.espr.org/app/uploads/EDiPR-outlineAugust-2021_08-2021.pdf Accessed (12 Oct 2021)

3. Education Committee (2021) European Diploma in Paediatric Radiology. Terms and Conditions. European Society of Paediatric Radiology. Available at https://www.espr.org/app/uploads/ EDiPR-Terms-and-Conditions_08-2021.pdf Accessed (12 Oct 2021)

4. European Board of Neuroradiology (2021) 2. Eligibility Criteria and Terms \& Conditions. European Diploma in Pediatric Neuroradiology. European Diplomas. European Board of Neuroradiology. Available at https://www.ebnr.org/en/european-diplomas/ european-diploma-in-pediatric-neuroradiology/2-eligibility-crite ria-and-terms-conditions/ Accessed (12 Oct 2021)

5. Junior ESPR (2021) Junior Grants. Educational Grants to Support Attendance to ECPR. European Society of Paediatric Radiology. Available at https://www.espr.org/junior-espr/ Accessed (12 Oct 2021)

6. European Society of Paediatric Radiology Events (2021) Junior Grants Application: ECPR 2021. European Society of Paediatric Radiology. Available at https://www.espr.org/news/events/exami nation-at-ecpr-2021-course-junior-grant-application-ecpr-2021/ Accessed (12 Oct 2021)

Publisher's note Springer Nature remains neutral with regard to jurisdictional claims in published maps and institutional affiliations. 